\title{
Cross-eyed - cross-spectral iris/periocular recognition database and competition
}

Conference or Workshop Item

Published Version

Creative Commons: Attribution-Noncommercial 3.0

Open Access

Sequeira, A., Chen, L., Ferryman, J., Alonso-Fernandez, F., Bigun, J., Raja, K., R., R., Christoph, B. and Peter, W. (2016) Cross-eyed - cross-spectral iris/periocular recognition database and competition. In: 2016 International Conference of the Biometrics Special Interest Group (BIOSIG), 21-23 Sept. 2016, Darmstadt, Germany. Available at http://centaur.reading.ac.uk/68957/

It is advisable to refer to the publisher's version if you intend to cite from the work. See Guidance on citing.

Published version at: https://doi.org/10.1109/BIOSIG.2016.7736915

All outputs in CentAUR are protected by Intellectual Property Rights law, including copyright law. Copyright and IPR is retained by the creators or other copyright holders. Terms and conditions for use of this material are defined in the End User Agreement. 


\section{www.reading.ac.uk/centaur}

\section{CentAUR}

Central Archive at the University of Reading

Reading's research outputs online 


\title{
Cross-Eyed - Cross-Spectral Iris/Periocular Recognition Database and Competition
}

\author{
Ana F. Sequeira ${ }^{1}$, Lulu Chen ${ }^{1}$, James Ferryman ${ }^{1}$, Fernando Alonso-Fernandez ${ }^{2}$, Josef Bigun ${ }^{2}$, \\ Kiran B. Raja ${ }^{3}$, R. Raghavendra ${ }^{3}$, Christoph Busch ${ }^{3}$, Peter Wild ${ }^{4}$ \\ ${ }^{1}$ School of Systems Engineering, University of Reading, Whiteknights, Reading, UK, \\ \{a.f.p.sequeira, 1.chen,j.m.ferryman\}@reading.ac.uk \\ ${ }^{2}$ Halmstad University, Sweden \{feralo, josef.bigun\}@hh.se \\ ${ }^{3}$ Norwegian Biometrics Laboratory, NTNU, Norway, \\ \{kiran.raja, raghavendra.ramachandra, christoph.busch\}@ntnu.no \\ ${ }^{4}$ AIT Austrian Institute of Technology GmbH, Austria, Peter.wild@ait.ac.at
}

\begin{abstract}
This work presents a novel dual-spectrum database containing both iris and periocular images synchronously captured from a distance and within a realistic indoor environment. This database was used in the $1^{\text {st }}$ Cross-Spectrum Iris/Periocular Recognition Competition (Cross-Eyed 2016). This competition aimed at recording recent advances in cross-spectrum iris and periocular recognition. Six submissions were evaluated for crossspectrum periocular recognition, and three for iris recognition. The submitted algorithms are briefly introduced. Detailed results are reported in this paper, and comparison of the results is discussed.
\end{abstract}

\section{INTRODUCTION}

The iris is well known as an accurate biometric trait. However, it is challenging to obtain sufficient quality for reliable recognition under less contrained acquisition. The periocular biometric trait, capturing the surrounding features of the eye region, facilitates incresead performance for low quality iris recognition and partial face recognition in less contrained conditions, such as from a distance or on the move.

Traditionally, commercial implementations of iris recognition systems use iris images acquired in the near infrared (NIR) spectrum. More recently, the use of noisy images captured in the visible (VIS) spectrum has atracted incresead interest. Highly pigmented irises present challenges when captured under VIS light mostly due to the pigmentation [1] but also due to specular reflections. Nevertheless, for less pigmented irises the VIS imaging can highlight the iris texture better than under NIR. This paper describes the first competition targeting cross-spectrum comparison as well as the benchmark dataset released for the competition. The key contributions of this work are: (1) A public database for crossspectral iris/periocular recognition. The database is distributed to academic and industry to support reproducible research. (2) Benchmark results on the database obtained through a public competition and showing trustworthy results due to the fact that methods were evaluated on a dataset unknown to participants.

The paper presents an overview of multispectral ocular recognition in Section II. The competition's database is introduced in Section III. Section IV lists results, which are discussed in more detail explaining methods in Section V. Finally, Section VI presents the conclusion.

\section{Multi-Spectral Recognition}

In the literature one can find works exploiting multispectral techniques from several perspectives. Joint NIR and VIS iris acquisition is patented by Burge and Monaco [2]. In [3] a method is proposed that aims for enhanced accuracy when information from NIR+VIS bands is combined. Ross et al. [4] proposed an enhanced iris recognition system by considering the fusion of multiple spectra beyond wavelengths of 900 $\mathrm{nm}$. Ngo et al. [5] give the design and implementation of a multispectral iris images acquisition system aimed at enhanced accuracy when information is combined. Another approach was proposed using the predictive NIR image based on the colour image and a slightly enhanced accuracy is reported when compared to matching the red channel only in [6]. Another work that studies the fusion of different spectra information and the cross-spectrum comparison is [7] where it is shown that cross-spectral performance presents itself as highly challenging (reporting EERs $>33 \%$ when comparing NIR against RGB channels across feature types).

Recently, Ramaiah and Kumar [8] exploit the cross-spectral comparison concluding that even though the comparisons in near infrared and visible channels are independently quite accurate, the performance of cross-spectral iris matching is significantly degraded. The results show how challenging this task is by reporting a value of $34 \%$ for equal error rate (EER) in the NIR to VIS comparison. The authors of the referred work, stress that there is a need to develop large-scale crossspectral iris databases and advanced algorithms to accurately match such images. If iris cross-spectrum comparison is still an open problem, periocular cross-spectrum comparisons is an even less studied task. Recently, a cross-spectral periocular matching technique has been explored in [9]. This work shows how existing algorithms fail in the cross-spectral comparison task and for the proposed method reports values below $50 \%$ for the verification accuracy at $1 \%$ false acceptance rate (FAR) in the NIR to VIS comparison. An even more challenging 
problem involves matching the ocular region of a face image obtained in the VIS spectrum with an iris image obtained in the NIR spectrum [10].

In the biometrics field, to allow advances and developments of new techniques, the existence of suitable databases is crucial. Multi-spectral databases already exist, however, not all the requisites for a realistic evaluation of the crossspectrum matching are met by them such as having images synchronously acquired or comprising both iris and ocular regions for further comparison. In [3] multi-spectral iris data is presented in which each iris snapshot outputs data acquired by four different spectral channels: NIR, R, G and B. The channels are perfectly registered and synchronized and can be used to form a false-colour image if those three channels are treated as traditional R, G, and B channels. The UTIRIS iris database [11] comprise NIR and VIS images of the same subjects however captured at different times with two different devices. More recently, Ramaiah and Kumar in [8] propose a new iris database composed by NIR and VIS images acquired simultaneously. The first cross-spectrum database with periocular images presented in [9] comprises images from three individual spectrums: VIS, night vision, and NIR.

\section{The CROSS-Eyed DATABASE}

The Reading Cross-Spectral Iris/Periocular Dataset (CrossEyed) $[12]^{1}$, was used as the benchmark dataset for the $1^{\text {st }}$ Cross-Spectral Iris/Periocular Competition. This dataset is composed by both VIS and NIR images captured with a custom developed dual spectrum imaging sensor, see Figure 1, which acquires NIR and VIS images synchronously. Using a hot mirror with $90 \%$ NIR reflectivity and $80 \%$ VIS transmission and a calibrated setup, images are captured by highresolution $(2 \mathrm{~K} \times 2 \mathrm{~K})$ machine vision cameras. The benefits for splitting beams before acquisition are the syncronous acquisition as well as a setup with standard components, however requiring a solid calibration of both cameras and lenses.

The images were captured from 120 subjects of several nationalities, ethnicities and eye colours. The ethnicity distribution is: caucasians $75 \%$, asians $17 \%$, indians $4 \%$ and africans $3 \%$. The age range is from 20 to 70 years old. The male/female distribution is $66 \% / 34 \%$.

The Cross-Eyed dataset is composed by 3 subsets: iris, masked periocular and ocular images obtained by manually cropping the face images. For each subset, 8 images of both eyes captured in both spectra were provided by each of the 120 individuals totalizing 3840 images for each. To avoid usage of iris information by periocular methods and to distinguish both tasks proposed, the periocular images have a mask on the whole eye region (as discussed in [13]). The periocular/ocular images are $900 \times 800$ pixels and the iris images are $400 \times 300$ pixels. Figure 1 illustrates some of the challenging conditions observed.

\footnotetext{
${ }^{1}$ Available by request at www.crosseyed.eu.
}

The main features of this database are: synchronised NIR + VIS images; images acquired from distance: 1.5 metre; uncontrolled realistic indoor environment; large variation in ethnicity and eye colour; and realistic and challenging illumination reflection. Table I shows a comparison between CrossEyed and other recent cross-spectrum ocular databases.

\section{THE CROSS-EYED COMPETITON}

The $1^{\text {st }}$ Cross-Spectrum Iris/Periocular Recognition Competition (Cross-Eyed 2016) covered two tasks: periocular and iris recognition and in both the main challenge was to perform the comparison step between NIR and VIS images. Besides the importance of releasing new datasets to the research community, this competition aimed at recording advances in cross-spectrum iris/periocular recognition and to connect different research groups working on this topic.

The Cross-Eyed 2016 competition received registrations from 13 participants from regions all over the globe. Among the registered participants, 4 participants submitted their algorithms: HH from Halmstad University, Sweden; NTNU from Norwegian Biometrics Laboratory, Norway; Aurora and Bmscians, BMSCE College, India (not included in this paper because the final methods were not compliant to the submission guidelines).

\section{A. HH periocular methods}

The HH system exploits the fusion of algorithms based on Symmetry Patterns (SAFE) [14], Gabor Spectral Decomposition (GABOR) [15], SIFT [16], Local Binary Patterns (LBP), and Histogram of Oriented Gradients (HOG) [13].

The matching scores from each matcher are mapped to a log-likelihood ratio according to the probabilistic Bayesian framework [17], to provide a fusion framework by simply summing individual scores that allows an efficient probabilistic interpretation of the resulting score. The fusion function is trained with scores obtained by matching images both from the same sensor and from different sensors, to cope with same and cross spectrum matchings. The $\mathrm{HH}$ team algorithms were based on different feature fusions: $H H_{1}$ (all five features), $\mathrm{HH}_{2}$ (SAFE+GABOR+LBP+HOG) and $\mathrm{HH}_{3}$ (GABOR+LBP+HOG).

\section{B. NTNU iris and periocular methods}

NTNU approach leverages the response images obtained from a bank of binarized statistical independent filters (BSIF). The BSIF filters learnt using natural images have proven their reliability in vast image classification problems [18]-[20]. Motivated by the earlier success of BSIF, NTNU employed multi-scale BSIF [20], [21] through an ensemble approach. To extract the iris region a coarse iris localisation technique based on [22] is employed. The responses from the MBSIF filters are represented in histograms that are compared using the $\chi^{2}$ distance metric. The obtained matching scores corresponding to each filter are again fused using a Support Vector Machine (SVM) classifier. Three algorithms submitted by NTNU (presented as $N T N U_{4}, N T N U_{5}$ and $N T N U_{6}$ ) 


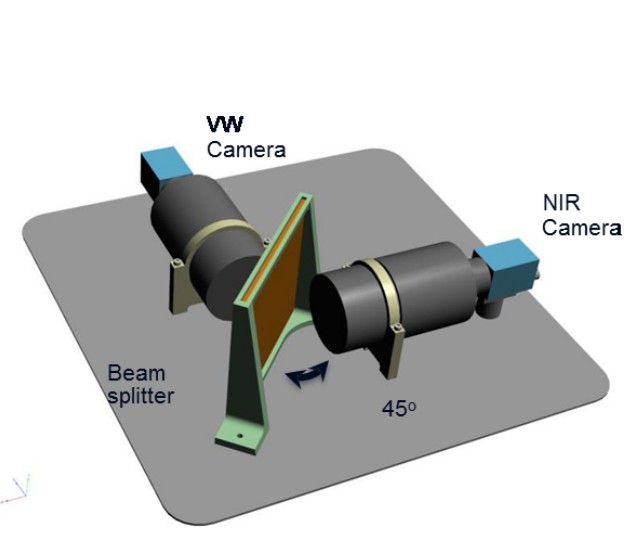

(a)

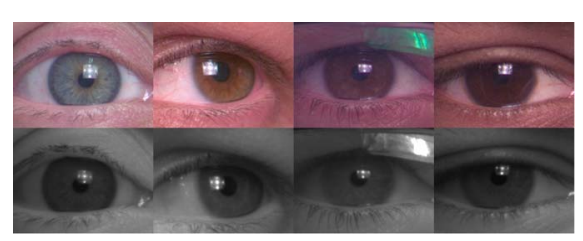

(b)

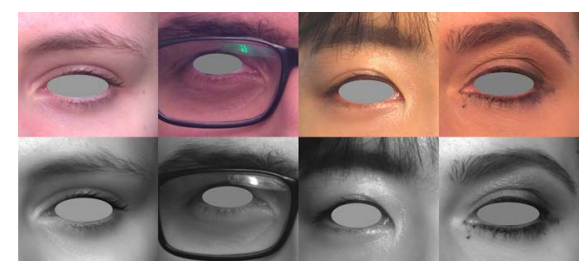

(c)

Fig. 1. Cross-Eyed VIS+NIR a) Sensor b) Iris images: Normal, Off-angle, Glasses reflections, Heavily pigmented. c) Periocular images: Normal, Glasses reflection, Hair occlusion, Heavy make-up.

TABLE I

MAIN FEATURES OF RECENT CROSS-SPECTRUM (NIR \& VIS) OCULAR DATABASES.

\begin{tabular}{ccccccc}
\hline $\begin{array}{c}\text { IITD Periocular } \\
{[9]}\end{array}$ & $\begin{array}{c}\text { VWU } \\
{[3]}\end{array}$ & $\begin{array}{c}\text { UTIRIS } \\
{[11]}\end{array}$ & $\begin{array}{c}\text { Hong Kong } \\
{[8]}\end{array}$ & $\begin{array}{c}\text { Cross-Eyed } \\
{[12]}\end{array}$ & $\begin{array}{c}\text { Iris/Ocular } \\
{[10]}\end{array}$ \\
\hline \hline ROI & Iris & Iris & Iris & Periocular & Iris, periocular, ocular Iris (i), ocular (o) \\
\hline Spectrum & NIR, VIS, night vis NIR, R/G/B NIR, VIS & NIR, VIS & synch. NIR, VIS & NIR (i), VIS (o) \\
\hline Users & 62 & 24 & 79 & 209 & 120 & 704 \\
\hline Images & 1240 & 480 & 3080 & 25080 & 11520 & 2716 \\
\hline
\end{tabular}

applied the same feature extraction technique for both iris and periocular, whereas the classifier employed in the comparator to obtain a fused score is learnt using the scores from three different training sets (combined training set, training set-2 and training set-1).

\section{EVALUATION, RESULTS AND DISCUSSION}

The evaluation comprised enrolment and template matching. Firstly, templates were obtained for all images. Then 2 types of comparisons were made: intra-class (every NIR image against each VIS image) and inter-class (3 NIR images against 3 VIS images, due to the computational effort of comparing all images).

To comply with the ISO/IEC standards [23], the generalized false accept rate (GFAR) and generalized false reject rate (GFRR) evaluation metrics were used. They generalize the false match rate (FMR) and the false non-match rate (FNMR) to include failure to acquire (FTA) and failure to enroll (FTE) rates, and are defined as: $G F A R=\alpha \times \beta \times F M R$ and $G F R R=F T E+\beta \times F T A+\alpha \times \beta \times F N M R$, where $\alpha=1-F T A$ and $\beta=1-F T E$. To compare the algorithms, the GFRR@GFAR=0.01 indicated as GF2 is used. The EER is reported to indicate the symmetrical accept and reject rates. Also the computational time for enrolment and matching is listed for each algorithm.

The final evaluation was made in a test dataset with 80 and 160 users for the periocular and iris tasks, respectively. Tables II and III reports the ranked performance and Figure 2 illustrates the DET curves of both periocular and iris algorithms.

\section{Periocular Recognition Results}

$H H_{1}$ achieved nearly $100 \%$ accuracy giving the best performance in periocular recognition. $\mathrm{HH}_{2}$ and $\mathrm{HH}_{3}$ had similar performance, but significantly lower than $H H_{1}$. It seems that SIFT features contribute decisively to the outstanding result in $H H_{1}$. However, the fusion in $H H_{1}$ is computationally heavy both in processing time and template size mainly due

TABLE II

PERFormance of PERIOCUlar METHOdS (GF2, EER, ENROLMENT TIME $T_{E}$ AND MATCHING TIME $\left.T_{M}\right)$.

\begin{tabular}{cccccc}
\hline Rank & Periocular Method & GF2 [\%] & EER [\%] & $\boldsymbol{T}_{\boldsymbol{E}}$ [seconds] & $\boldsymbol{T}_{\boldsymbol{M}}$ [seconds] \\
\hline \hline 1 & $H H_{1}$ & 0.00 & 0.29 & 18.05 & 0.70 \\
\hline 2 & $H H_{2}$ & 9.14 & 5.24 & 14.40 & 0.05 \\
\hline 3 & $H H_{3}$ & 11.42 & 6.02 & 14.39 & 0.05 \\
\hline 4 & $N T N U_{1}$ & 14.43 & 4.84 & 6.03 & 5.15 \\
\hline 5 & $N T N U_{2}$ & 14.71 & 6.48 & 6.03 & 5.18 \\
\hline 6 & $N T N U_{3}$ & 33.38 & 22.28 & 6.03 & 5.16 \\
\hline
\end{tabular}

TABLE III

PERFormance OF IRIS METHOdS (GF2, EER, ENROLMENT TIME $T_{E}$ AND MATCHING TIME $T_{M}$ ).

\begin{tabular}{cccccc}
\hline Rank & Iris Method & GF2 [\%] & EER [\%] & $\boldsymbol{T}_{\boldsymbol{E}}$ [seconds] & $\boldsymbol{T}_{\boldsymbol{M}}$ [seconds] \\
\hline \hline 1 & $N T N U_{6}$ & 3.31 & 2.78 & 118.68 & 5.15 \\
\hline 2 & $N T N U_{5}$ & 3.34 & 2.83 & 118.68 & 5.14 \\
\hline 3 & $N T N U_{4}$ & 3.62 & 3.11 & 118.68 & 5.15 \\
\hline
\end{tabular}




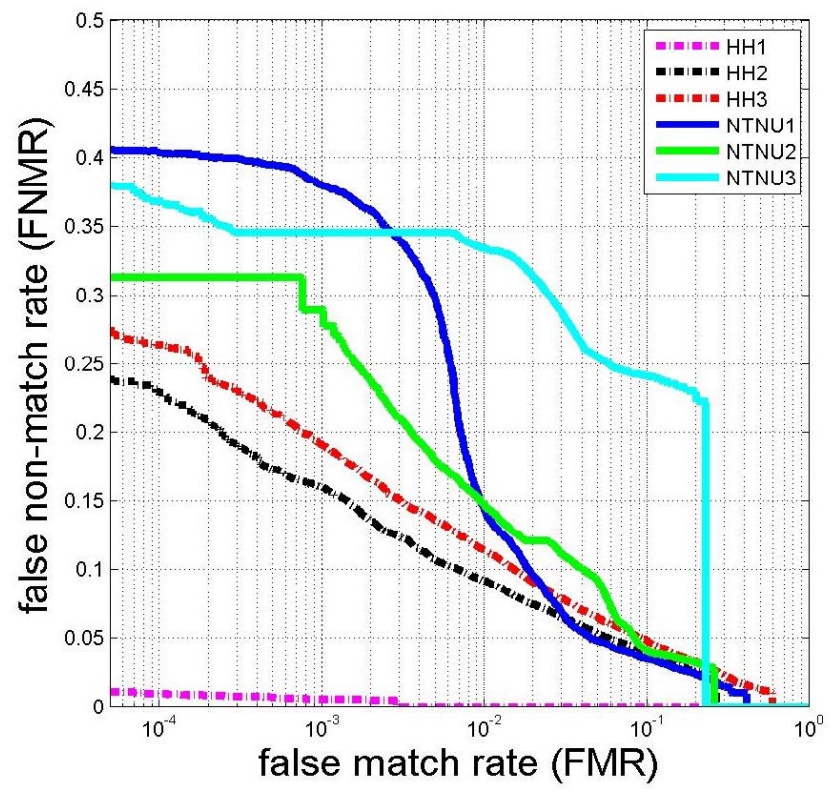

(a)

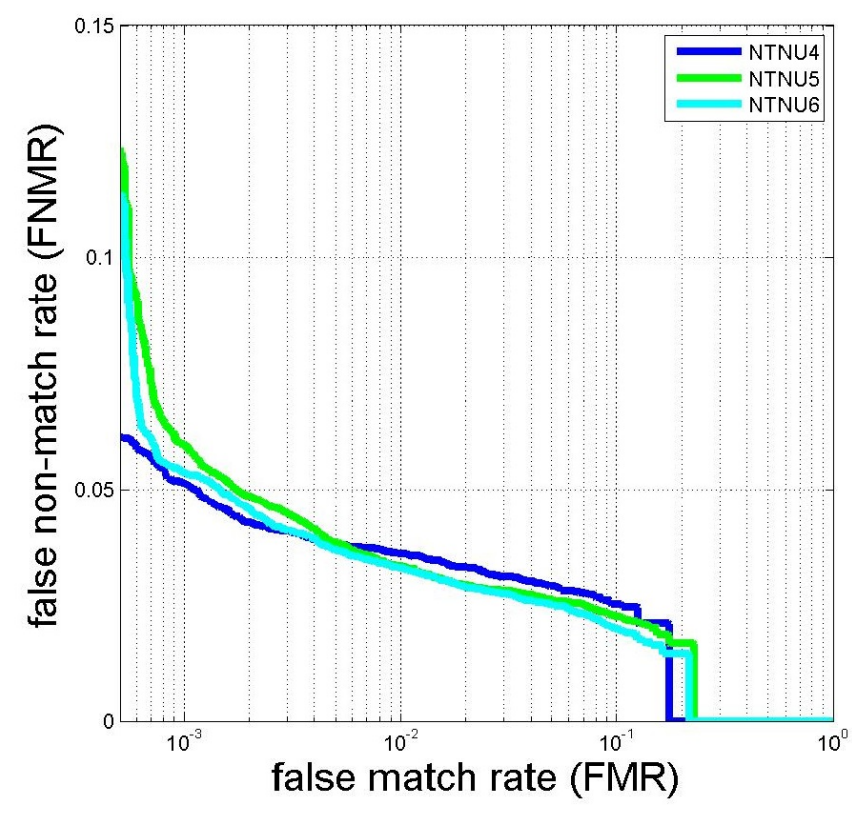

(b)

Fig. 2. DET curves of the: (a) periocular methods (b) iris methods.

to the SIFT process. HH's fusion algorithms performed relatively better than the learning-based methods from the NTNU, however $N T N U_{1}$ and $N T N U_{2}$ provided a good performance similar to $\mathrm{HH}_{3}$. The observable difference in $\mathrm{NTNU}_{3}$ indicates the generalisation issue when applied to large-scale comparisons, which can be attributed to the comparison score level fusion using SVM. The model learnt on the training set is not robust enough to handle the data in large scale comparisons where the scores vary to a greater extent. The GF2 rates from all algorithms showed good performance. Even not recalling the $H_{1}$ outstanding result, the other methods provided GF2 values between $9 \%$ and $33 \%$. Even the highest value is below the state-of-the-art result of $53 \%$ reported in [9].

\section{Iris Recognition Results}

All three NTNU algorithms for iris recognition achieved very good results, where both error rates (GF2 and EER) were lower than 4\%. A recent state-of-the-art work [8] showed how challenging this task is by reporting a value of $34 \%$ for EER in the NIR to VIS comparison of iris images. High rate of accuracy in verification can be attributed to the block based approach employed.

The iris algorithms are the same as the periocular algorithms. Hence, it is interesting to observe how the iris performance compares to the periocular performance. All three iris recognition systems outperformed periocular verification for NTNU team. Although the iris images from this dataset present challenging factors, the iris patterns preserve highly discriminate information compared to the periocular region, even for cross-spectral comparison. Further, leveraging the information from the red channel alone in the visible spectrum has influence in achieving high verification rates for cross- spectral iris matching.

However, the processing time for generating the template in the iris is more demanding in terms of computation time than that for the periocular task. One key factor in such a time response is due to localisation of iris followed by segmentation which are both complex tasks in VIS images.

\section{CONCLUSIONS}

This paper presents the first competition on cross-spectral periocular and iris recognition and its benchmark dataset. This competition and dataset should provide valuable resources to the biometric research community and promote this relatively new topic. The use of this database will prevail beyond the competition, giving an opportunity for creating spectrumindependent algorithms and evaluating them in experiments. Overall, the cross-spectrum comparison posed a serious challenge to the participants. The results show beyond doubt that this problem is not fully investigated and there is room for improvement. Most of the methods have achieved good results, especially the fusion based $H H_{1}$ algorithm that obtained a nearly $100 \%$ accuracy. The learning-based algorithms showed outstanding performance on the iris recognition, but they also showed problems in generalizing to large-scale comparisons. In a broader view, this initiative contributed to the usability of iris/periocular recognition technologies on generic devices and diverse scenarios.

\section{ACKNOWLEDGMENTS}

This work is supported by: the EU FastPass project (grant agreement 312583), the Swedish Research Council and the CAISR program of the Swedish Knowledge Foundation, the Research Council of Norway (grant IKTPLUSS 248030/O70). 


\section{REFERENCES}

[1] A. Ross, "Iris recognition: The path forward," Computer, vol. 43, no. 2, pp. 30-35, Feb 2010.

[2] M. J. Burge and M. K. Monaco, "Multispectral iris fusion for enhancement, interoperability, and cross wavelength matching," in Proc. SPIE, vol. 7334,2009 , p. 8.

[3] C. Boyce, A. Ross, M. Monaco, L. Hornak, and X. Li, "Multispectral iris analysis: A preliminary study," in Proc. CVPRW, 2006, p. 12.

[4] A. Ross, R. Pasula, and L. Hornak, "Exploring multispectral iris recognition beyond 900nm," in Proc. BTAS, 2009, pp. 1-8.

[5] H. Ngo, R. Ives, J. Matey, J. Dormo, M. Rhoads, and D. Choi, "Design and implementation of a multispectral iris capture system," in Proc. ACSSC, 2009, pp. 380-384.

[6] J. Zuo, F. Nicolo, and N. A. Schmid, "Cross spectral iris matching based on predictive image mapping," in Proc. BTAS. IEEE, 2010, pp. 1-5.

[7] P. Wild, P. Radu, and J. Ferryman, "On fusion for multispectral iris recognition," in Proc. ICB, May 2015, pp. 31-37.

[8] N. P. Ramaiah and A. Kumar, "Advancing cross-spectral iris recognition research using bi-spectral imaging," in Mach. Intell. and Signal Proc. Springer, 2016, pp. 1-10.

[9] A. Sharma, S. Verma, M. Vatsa, and R. Singh, "On cross spectral periocular recognition," in Proc. ICIP. IEEE, 2014, pp. 5007-5011.

[10] R. Jillela and A. Ross, "Matching face against iris images using periocular information," in Proc. ICIP, 2014, pp. 4997-5001.

[11] M. S. Hosseini, B. N. Araabi, and H. Soltanian-Zadeh, "Pigment melanin: Pattern for iris recognition," IEEE Trans. Instr. Measur., vol. 59, no. 4, pp. 792-804, April 2010.

[12] A. F. Sequeira, L. Chen, P. Wild, P. Radu, and J. Ferryman, "Cross-Eyed: Reading Cross-Spectrum Iris/Periocular Dataset," 2016, www.crosseyed.eu.

[13] U. Park, R. Jillela, A. Ross, and A. K. Jain, "Periocular biometrics in the visible spectrum," IEEE TIFS, vol. 6, no. 1, pp. 96-106, 2011.

[14] A. Mikaelyan, F. Alonso-Fernandez, and J. Bigun, "Periocular recognition by detection of local symmetry patterns," in Proc IEB-SITIS, 2014, pp. 584-591.

[15] F. Alonso-Fernandez and J. Bigun, "Near-infrared and visible-light periocular recognition with gabor features using frequency-adaptive automatic eye detection," IET Biometrics, vol. 4, no. 2, pp. 74-89, 2015.

[16] F. Alonso-Fernandez, P. Tome-Gonzalez, V. Ruiz-Albacete, and J. Ortega-Garcia, "Iris recognition based on SIFT features," in Proc. BIDS, 2009, pp. 1-8.

[17] F. Alonso-Fernandez, J. Fierrez, D. Ramos, and J. Ortega-Garcia, "Dealing with sensor interoperability in multi-biometrics: the UPM experience at the biosecure multimodal evaluation 2007," in Proc. SPIE BTHI, vol. 6944, 2008, p. 12.

[18] J. Kannala and E. Rahtu, "BSIF: Binarized statistical image features," in Proc. ICPR. IEEE, 2012, pp. 1363-1366.

[19] Kiran B. Raja, R. Raghavendra, M. Stokkenes, and C. Busch, "Multimodal authentication system for smartphones using face, iris and periocular," in Proc. ICB, 2015, pp. 143-150.

[20] R. Raghavendra and C. Busch, "Robust scheme for iris presentation attack detection using multiscale binarized statistical image features," IEEE TIFS, vol. 10, no. 4, pp. 703-715, 2015.

[21] Kiran B. Raja, R. Raghavendra, and C. Busch, "Binarized statistical features for improved iris and periocular recognition in visible spectrum," in Proc. IWBF, 2014, pp. 1-6.

[22] Kiran B. Raja, R. Raghavendra, V. K. Vemuri, and C. Busch, "Smartphone based visible iris recognition using deep sparse filtering," Pattern Rec. Lett., vol. 57, no. 0, pp. 33 - 42, 2015.

[23] International Organization for Standardization, "ISO/IEC 19795-1:2006 - Biometric performance testing and reporting - Part 1: Principles and framework," 2006, rev. 2011. 